\title{
Excision Repair Cross-Complementation Group 1 (ERCC1): A Prognostic and Predictive Biomarker in Patients with Colorectal Cancer Receiving Adjuvant Oxaliplatin Based Chemotherapy
}

\author{
Adel Gabr ${ }^{1 *}$, T. M. Elsaba², Khalid Razek ${ }^{3}$, Shaima Tamam ${ }^{4}$, Haisam Atta ${ }^{5}$ \\ ${ }^{1}$ Department of Medical Oncology, South Egypt Cancer Institute, Assuit University, Assiut, Egypt \\ ${ }^{2}$ Department of Pathology, South Egypt Cancer Institute, Assuit University, Assiut, Egypt \\ ${ }^{3}$ Department of Surgical Oncology, South Egypt Cancer Institute, Assuit University, Assiut, Egypt \\ ${ }^{4}$ Department of Radiotherapy, South Egypt Cancer Institute, Assuit University, Assiut, Egypt \\ ${ }^{5}$ Department of Diagnostic Radiology, South Egypt Cancer Institute, Assuit University, Assiut, Egypt \\ Email: ^adelgabre@yahoo.com, adelgabr@aun.edu.eg,shimaa@aun.edu.eg, haisamasa@yahoo.com, \\ tarek_elsaba@aun.edu.eg,rezk.khalid@gmail.com
}

How to cite this paper: Gabr, A., Elsaba, T.M., Razek, K., Tamam, S. and Atta, H. (2016) Excision Repair Cross-Complementation Group 1 (ERCC1): A Prognostic and Predictive Biomarker in Patients with Colorectal Cancer Receiving Adjuvant Oxaliplatin Based Chemotherapy. Journal of Cancer Therapy, 7, 622-634. http://dx.doi.org/10.4236/jct.2016.79065

Received: June 14, 2016

Accepted: August 29, 2016

Published: September 1, 2016

Copyright $\odot 2016$ by authors and Scientific Research Publishing Inc. This work is licensed under the Creative Commons Attribution International License (CC BY 4.0).

http://creativecommons.org/licenses/by/4.0/

\section{Abstract}

Background: Colorectal cancer (CRC) ranks as the third most common cancer and the third most killing cancer worldwide [1]. The addition of oxaliplatin to fluorouracil (FOLFOX) or capecitabine (CAPOX) has become a fundamental component of chemotherapeutic regimens and chemoradiation in adjuvant treatment of CRC cancer. Excision repair cross-complementation group 1 (ERCC1) encodes an enzyme that is essential for the efficient repair of DNA damage induced by platinum compounds including Oxaliplatin. Purpose: This study aims to investigate the role of ERCC1 as a predictive and prognostic marker in colorectal patients receiving oxaliplatin based chemotherapy and chemoradiation. Patients and Methods: 100 annotated stage III CRC patients were prepared as immunohistochemical (IHC) analysis of ERCC1 protein expression. All of the patients received oxaliplatin based chemotherapy. Results: Analysis of data showed that high ERCC1 expression was significantly associated with early treatment failure and disease free survival among patient with stage III CRC. Conclusion: High ERCC1 expression was an independent predictor factor of early treatment failure $(P<0.018)$ and associated with lower disease free survival $(P=0.004)$.

\section{Keywords}

ERCC1, CRC, Oxaliplatin, IH 


\section{Introduction}

Colorectal cancer (CRC) ranks as the third most common cancer and the third most killing cancer worldwide [1]. It is estimated that around 608,000 deaths from CRC worldwide, accounting for $8 \%$ of global cancer-related mortalities [2]. Surgical resection remains to be the potentially curative option for CRC, but postoperative tumor relapse and distantmetastases greatly undermine the long-term survival outcome [3]. Although there is a dramatic improvement of chemotherapy, radiotherapy, and molecular targeted therapy, the addition of oxaliplatin to fluorouracil (FOLFOX) or capecitabine (CAPOX) has become a fundamental component of chemotherapeutic regimens in the standard adjuvant treatment of CRC [3]. MOSAIC trial clarified a 3-year DFS-improvement of FOLFOX4 superior to infusional fluorouracil and leucovorin; nevertheless, a recent update of the MOSAIC trial suggests that with further follow-up, the benefit of oxaliplatin is not of the magnitude that was expected [2] [4]. The recurrence of CRC is a time-dependent phenomenon and $60 \%-80 \%$ of recurrences become apparent within the first 2 years after initial resection [5]. CRC recurrences are mainly attributed to tumor characteristics (e.g., unfavorable genotype, deeper tumor invasion, lymph node metastasis, and advanced cancer stage) [6]. Even though patients with the same TNM stage (The TNM system is the most widely used cancer staging system. Most hospitals and medical centers use the TNM system as their main method for cancer reporting) may display observable variation in clinical outcome and response rates (RRs) to treatment, elucidating that the conventional staging procedures may not accurately predict prognosis [3]. Therefore, it is of utmost importance to find out new molecular markers that will help us to reclassify the patients as regards their clinical prognosis as well as sensitivity to chemotherapy in order to tailor their management strategy [7]. Oxaliplatin, a cytotoxic agent from the diaminocyclohexane platinum family, exerts its cytotoxic effects through the formation of DNA adducts that block both DNA replication and transcription in actively dividing cells [8]. Meanwhile, some authors believed that the increased removal of bulky DNA adducts, which can also be produced by the other detrimental carcinogenic factors, is beneficial for the genome stability and cancer prevention [9]. These adducts are recognized and repaired by the nucleotide excision repair (NER) pathway. The ERCC1 protein which is a major component of nucleotide excision repair (NER) pathway [10], not only involves the repair of interstrand cross-links in the DNA, but also involves the recognition and remove of cytotoxic agents like oxaliplatin [11]. The relationship between ERCC1 expression and clinical outcome has been investigated in various kinds of malignant cancers including gastric cancer [12], ovarian cancer [13], and lung cancer [14]. For patients with CRC, the definite prognostic value of ERCC1 expression has not been established yet. Some authors suggested that increased expression of ERCC1 predicted inferior RRs to chemotherapy and survival outcome [15]. While some other authors didn't find any significant association between ERCC1 expression level and clinical prognosis [16]. According to the previous clinical data, this study aims to investigate the prognostic and predictive role of ERCC1 expression among patients with stage III CRC receiving oxaliplatin based chemotherapy. 


\section{Patients and Methods}

\subsection{Patient Population}

Between January 2013 and April 2016, a total of one hundred patients with stage III colorectal cancer treated with radical surgery and received adjuvant treatment at South Egypt Cancer Institute were recruited to participate in this study after approval of the local ethics committee and patient's consent.

\subsection{Inclusion Criteria}

Patients of both gender, aged $\geq 18$ years with histologically confirmed colorectal adenocarcinoma; stage III according to American Joint Committee on Cancer and the Union for International Cancer Control (AJCC-UICC); 7th Edition. ECOG performance state $\leq 2$, adequate hematological (evidenced by white blood cell count $\geq 4000 / \mu \mathrm{l}$ and platelet count $\geq 100,000 / \mu \mathrm{l}$ ), renal (creatinine $<1.5 \mathrm{mg} / \mathrm{dl}$ ) and hepatic functions (serum total bilirubin $<1.5 \mathrm{mg} / \mathrm{dl}$ ). Characteristics of enrolled patients are shown in Table 1.

\subsection{Exclusion Criteria}

Patients with inadequate organ functions, and serious uncontrolled concomitant disease that would contraindicate the use of any of the chemotherapy drugs or interfere with cycle regularity were excluded from the study.

\subsection{Work-Up}

A series of 100 patients of stage III CRC, treated with radical surgey (Table 1). The routine diagnostic work-up including, history and physical examination. CT scans of chest, abdomen and pelvis before starting treatment. Blood sampling for complete blood count, renal and hepatic functions. Serum level of tumor marker CEA.

Response evaluation for oncological imaging employed Response evaluation criteria in solid tumors (RECIST) version 1.1.

\section{Treatment Schedule}

\subsection{Chemotherapy}

All the patients were treated with the standard FOLFOX-4 consisting of 2-hour intravenous infusion of oxaliplatin $\left(85 \mathrm{mg} / \mathrm{m}^{2}\right)$ on day 1 , and 2-hour intravenous drip infusion of calcium folinate $\left(200 \mathrm{mg} / \mathrm{m}^{2}\right)$ on days $1-2$, followed by intravenous injection of 5 -FU $\left(400 \mathrm{mg} / \mathrm{m}^{2}\right)$ and continuous infusion of 5-FU $\left(600 \mathrm{mg} / \mathrm{m}^{2}\right)$ lasting $22 \mathrm{~h}$ on days 1 2 , every 2 weeks. The toxic effects were evaluated using the national cancer institute common toxicity criteria [17]. According to the standard practice of our institution, in case of any grade III toxicity; treatment was interrupted until recovery and then restarted with a $25 \%$ dose reduction. Treatment was permanently stopped in case of any grade IV toxicity.

\subsection{Radiotherapy}

During concurrent chemoradiation for rectal cancer, chemotherapy protocol modified 
Table 1. The demographic characteristics of the 100 colorectal cancer patients in the study.

\begin{tabular}{|c|c|c|}
\hline Variable & Category & No. (\%) \\
\hline \multirow[t]{3}{*}{ Age } & $<40$ & $47(47 \%)$ \\
\hline & $40-50$ & $33(33 \%)$ \\
\hline & $>50$ & $20(20 \%)$ \\
\hline \multirow[t]{2}{*}{ Performance } & $<1$ & $75(75 \%)$ \\
\hline & 2 & $25(25 \%)$ \\
\hline \multirow[t]{2}{*}{ Gender } & Male & $52(52 \%)$ \\
\hline & Female & $48(48 \%)$ \\
\hline \multirow[t]{2}{*}{ tumor site } & Colon & $74(74 \%)$ \\
\hline & Rectum & $26(26 \%)$ \\
\hline \multirow[t]{7}{*}{ Histopathology } & Classic adenocarcinoma & $86(86 \%)$ \\
\hline & G1 & $24(27.9 \%)$ \\
\hline & G2 & $32(37.2 \%)$ \\
\hline & G3 & $30(34.8 \%)$ \\
\hline & Variant of adenocarcinoma & $14(14 \%)$ \\
\hline & Mucinous & $9(64.3 \%)$ \\
\hline & Signet ring carcinoma & $5(35.7 \%)$ \\
\hline \multirow[t]{2}{*}{ CEA } & Normal & $58(58 \%)$ \\
\hline & High & $42(42 \%)$ \\
\hline \multirow[t]{10}{*}{ Type of surgery } & RT hemicolectomy & $25(25 \%)$ \\
\hline & Lt Hemicolectomy & $20(20 \%)$ \\
\hline & Extended Rt hemicolectomy & $12(12 \%)$ \\
\hline & Extended LT hemicolectomy & $10(10 \%)$ \\
\hline & Near total colectomy & $4(4 \%)$ \\
\hline & Hartman's operation & $3(3 \%)$ \\
\hline & High anterior resection of Dixon & $9(9 \%)$ \\
\hline & Low anterior resection of Dixon & $10(10 \%)$ \\
\hline & Coloanal anastomosis with covering ileostomy & $3(3 \%)$ \\
\hline & Abdominoperineal resection & $4(4 \%)$ \\
\hline \multirow[t]{2}{*}{ ERCC1 expression } & High & $21(21 \%)$ \\
\hline & Low & $79(79 \%)$ \\
\hline
\end{tabular}

from classic FOLFOX to Oxaliplatin $50 \mathrm{mg} / \mathrm{m}^{2}$ day 1, 8, 22, 29 with Capecitabine 825 $\mathrm{mg} / \mathrm{m}^{2}$ PO bid on days 1 - 14 and $22-35$ (according to O'Connell et al., 2014 and Hofheinz et al., 2012).

Clinical target volume high risk (CTV-HR) includes remaining rectum, mesorectal bed, and presacral space, CTV-standard risk includes mesorectum bed and right and left internal iliac lymph nodes, external iliac lymph nodes for T4 tumors and perineals- 
pacein cases had abdominoperineal resection. Each CTV expanded $1 \mathrm{~cm}$ to form Planning target volume (PTV). Postoperative dose to PTV-HR: 54 Gy PTV-SR (Planning target volume standard risk): 45 Gy at $1.8 \mathrm{~Gy} /$ fraction.

\subsection{Follow-Up}

The duration of follow-up was defined as the time between surgery and disease recurrence, death or last hospital contact. The main target of follow up to detect early treatment failure (disease recurrence). History and physical examination every 3 months/ 2 years, then every 6 months later on. CEA level every 3 months/2 years then every 6 months later on. Chest/abdomen/pelvis CT every 6 months/2 years then every year later on. Colonoscopy every year.

\subsection{Immunohistochemically (IHC) Staining}

Briefly, four $\mu \mathrm{m}$ thick sections of each patient's sample were deparaffinised and rehydrated. The antigen retrieval for ERCC1 was performed with $0.01 \mathrm{~mol} / \mathrm{l}$ citrate buffer ( $\mathrm{pH}$ 6.0) in an $800 \mathrm{~W}$ microwave for 12 minutes. After blocking of endogenous peroxidase and non-specific reactions, sections were incubated for an hour at room temperature with diluted primary antibody (ERCC1 1/200; Primary mouse monoclonal ERCC-1 antibody (Clone 8F1) Novus biologicals). Ultra Vision Detection System Anti-Polyvalent, HRP/DAB (Ready-To-Use) [LAB VISION Corporation, catalogue \#TP-015-HD, Fremont, California 94539-6406, USA] was used as visualization system following manufacturer's instructions. Immunohistochemical staining was developed using diaminobenzidine $(\mathrm{DAB})$ as the substrate for 5 minutes. Sections then counterstained in Mayer's hematoxyline, dehydrated and mounted with DPX. Sections from Human tonsils used as positive control as recommended by the manufacturer datasheet. ERCC-1 positivity was identified as brownish nuclear staining [18].

\subsection{Evaluation of Immunohistochemistry}

The immunoreactivity was evaluated by a single pathologist without prior knowledge of the clinicopathological features and outcome data. The proportion of cells with ERCC1 expression was rated as follows: 0 points for $<5 \%$ positive tumor cells; 1 point for $5 \%$ $25 \%$ positive cells; 2 points for $26 \%-50 \%$ positive cells; 3 points for $51 \%-75 \%$ positive cells; and 4 points for $>75 \%$ positive cells, and the staining intensity graded as 0 for no staining, 1 for weak staining, 2 for moderate staining, and 3 for strong staining. The specimens were categorized into two groups according the overall immunoreactivity score (IRS) score into: ERCC1 negative ( 0 - 1 point), and ERCC1 positive ( $\geq 2$ points) as previously described [19], (Figure 1 and Figure 2).

\subsection{Statistical Analysis}

All statistical analyses were performed using SPSS version 20.0 (SPSS Inc., Chicago, IL). Data were described as frequencies (percentages). Differences in distributions between the variables examined were analyzed by chi-square test. Early failure was defined as 

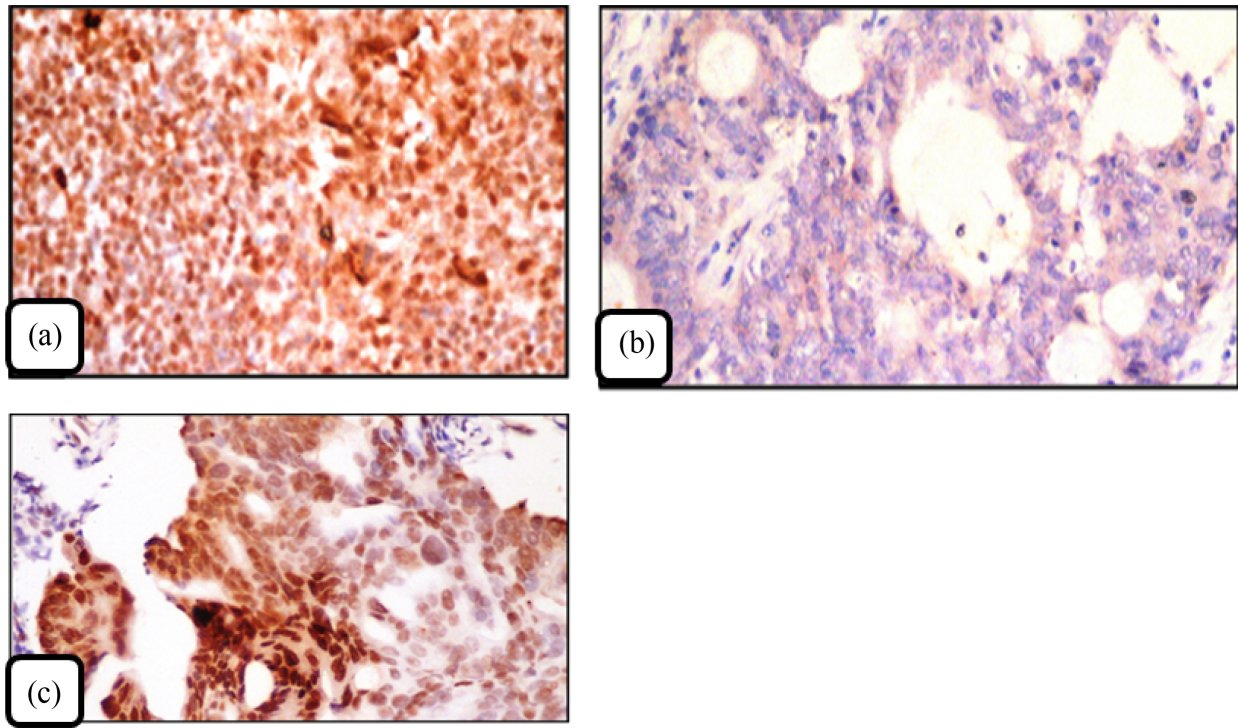

Figure 1. Immunohistochemical staining of ERCC1 in colorectal tumor tissue. ERCC1 expressed as a brown nuclear staining. (a) Human tonsil, as a positive control; (b) Cancer colon tissue show negative expression; (c) Cancer colon tissue show positive nuclear staining of ERCC1 expression.

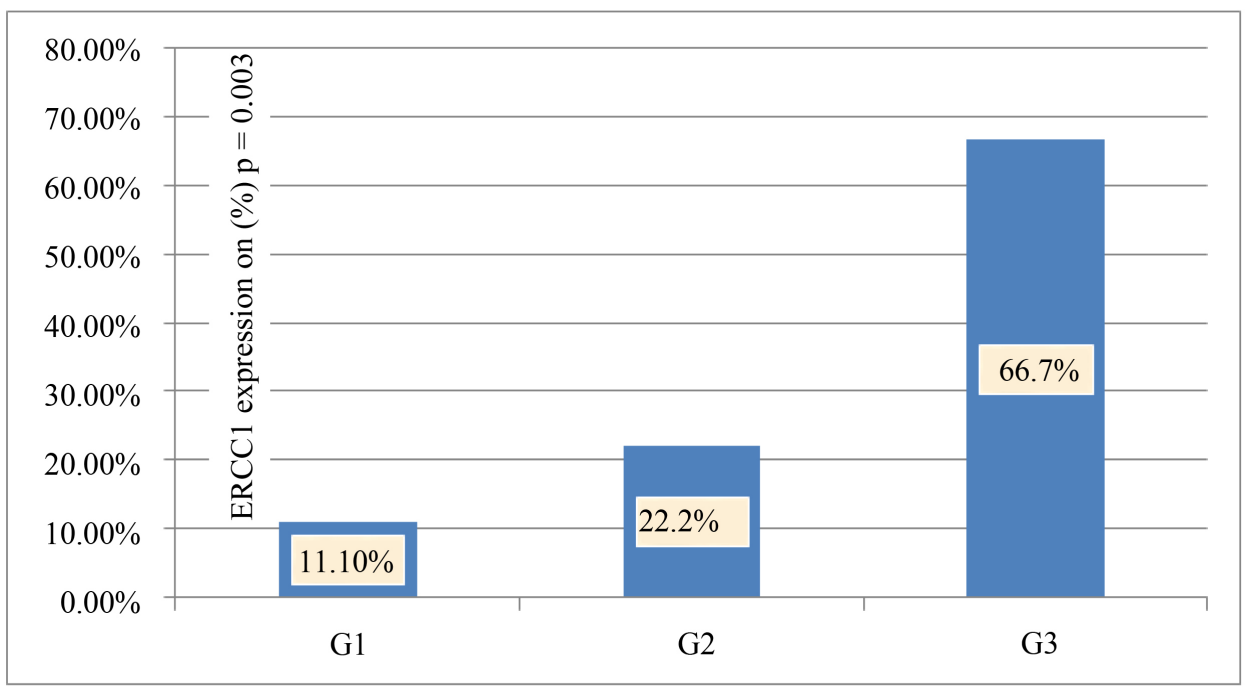

Figure 2. ERCC1 expression according to different grades of adenocarcinoma.

any local recurrence or distant metastases within the first 2 years after initial resection. DFS, defined as the time between the date of surgery and the first event (local or distant disease recurrence) or last follow up. PFS was defined as the time from the start of treatment to the time of the first record of progression or to the date of death. OS was assessed, in patients with stage IV, as the time from the initiation of first-line chemotherapy to death from any cause or last follow-up. Survival curve was estimated with the Kaplan-Meier method and compared using the log-rank test. A multivariate logistic regression analysis included all relevant clinicopathological variables, whatever their univariate P-value, namely, age, gender, differentiation grade, CEA level and ERCC1 
expression was performed by a forward stepwise method. A P-value $<0.05$ was considered statistically significant.

\section{Results}

1) The demographic data of 100 patients with stage III CRC are shown in (Table 1). The median age at diagnosis was 45 years (range, 19 - 69 years) with mean $41.2 \pm 12.3$. We followed the patients until April 2016 with a median follow-up period of 21 months (range, 6 - 32 months). TNM staging are shown in (Table 2).

2) ERCC1 protein expression: ERCC1 high expression was observed in 21 cases (21\%) while 79 cases (79\%) showed low ERCC1 expression. ERCC1 expression was significantly correlated with high histologic grade $(\mathrm{p}=0.003)$. ERCC1 was positive in only $2(9.5 \%)$ case of G1 adenocarcinoma tumors, 4 (19\%) cases of G2, and $12(57 \%)$ cases of G3. Association between ERCC1 expression and different grades of differentiation are shown in (Figure 3 and Figure 4). Regarding other pathologic types of adenocarcinoma, 3 cases (14.5\%) of mucinous tumors were positive for ERCC1, while all signet ring carcinoma tumors were negative for ERCC1.

Table 2. TNM staging of the stage III CRC patients in the study.

\begin{tabular}{ccc}
\hline Variable & Category & $\mathbf{N}=\mathbf{1 0 0}(\mathbf{\%})$ \\
\hline Tumor invasion (T) & $\bullet \mathrm{T} 2$ & $14(14 \%)$ \\
& $\bullet \quad \mathrm{T} 3$ & $70(70 \%)$ \\
& $\bullet \quad \mathrm{T} 4$ & $52(16 \%)$ \\
Lymph node (N) & $\bullet \mathrm{N} 1$ & $48(48 \%)$ \\
\hline
\end{tabular}

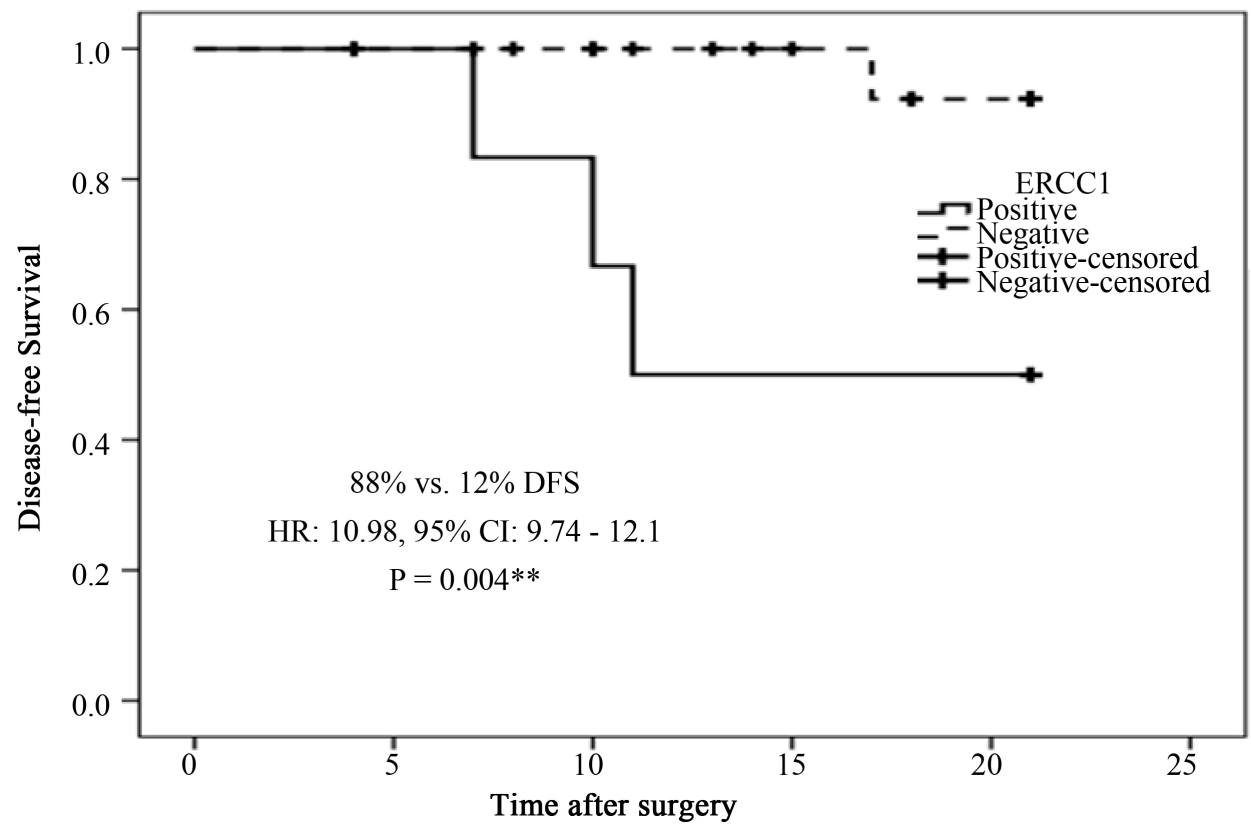

Figure 3. Relation between positive ERCC1 and DFS. 


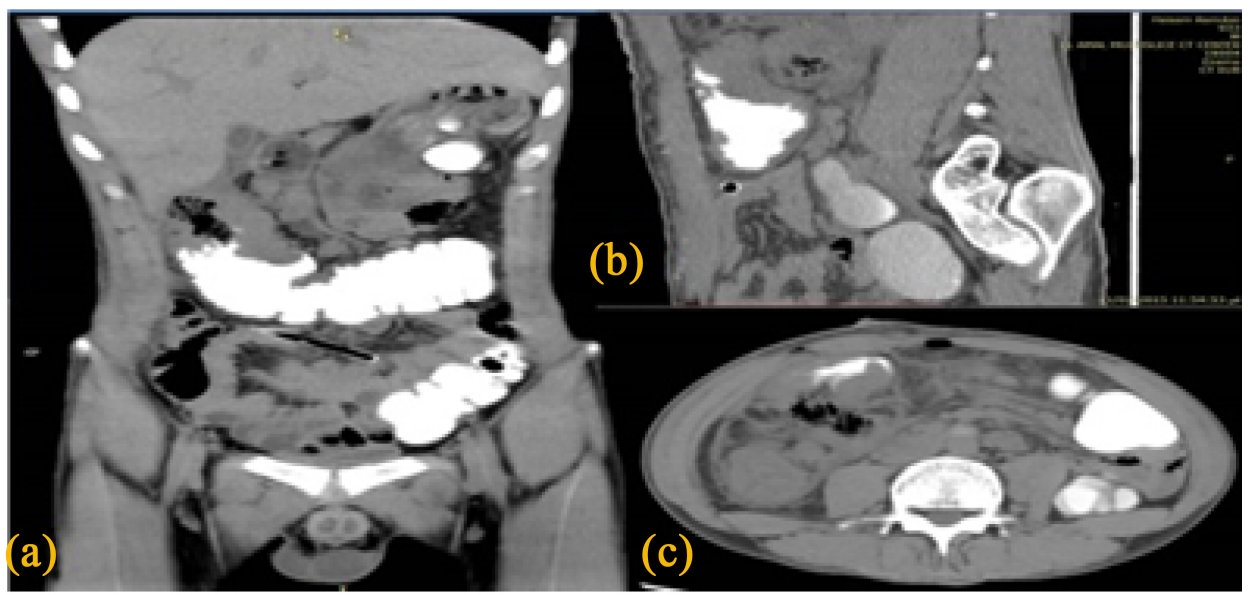

Figure 4. MDCT with enema demonstrating annular stricture colonic mass at hepatic flexure (a) Coronal scan; (b) Sagittal scan; and (c) Axial scan.

3) Pattern of treatment related toxicity: Concerning the main treatment side effects; neurotoxicity, GIT toxicity (vomiting/diarrhea) and myelosuppression appeared in $27 \%, 26 \%$, and $25 \%$ patients, respectively. Most cases were grades 1 or 2 . Hepatotoxicity was observed in $8(8 \%)$ patients. 8 patients were irregularly on treatment due to grade 2 - 3 toxicity. Oxaliplatin and 5-FU doses were both reduced by $25 \%$ in 5 patients with stage III due to grade III toxicity and treatment stopped in 2 cases due to grade IV toxicity.

\subsection{Association between Postoperative Early Failure and Clinicopathologic Features and ERCC1 in the Stage III CRC Patients}

1) Univariate analysis revealed that deeper tumor invasion $(P<0.002)$, high postoperative CEA level $(\mathrm{P}<0.041)$ and positive ERCC1 $(\mathrm{P}<0.004)$ were significantly associated with early treatment failure (Table 3 ). However, there was no significant association between gender, age, tumor location, histopathology or lymph node metastases and early treatment failure.

2) A multivariate logistic regression analysis using forward stepwise method revealed that ERCC1 expression (HR: 0.045; 95\% CI, $1.1-2.4$; P $<0.018$ ), deeper tumor invasion (HR, 1.42; 95\% CI, $0.003-0.57$; P 0.001) and high postoperative CEA level (HR, 9.5; 95\% CI, 0.83 - 109.2; P < 0.041) were an independent predictors for early failure following FOLFOX-4 adjuvant chemotherapy (Table 4 and Table 5).

\subsection{Survival Analysis of ERCC1 Expression in Stage III CRC Patients}

The DFS in CRC patients with high ERCC1 expression was significantly shorter than that in CRC patients with low ERCC1 expression (16 vs. 25 months; $\mathrm{P}=0.004$; Figure 2).

\section{Discussion}

Combination Oxaliplatin and 5-FU is a standard chemotherapy regimen in stage III 
Table 3. The main side effects of chemotherapy.

\begin{tabular}{ccccccc}
\hline Side-effects & Anemia & Neutropenia & Thromcytopenia & GIT-toxicity & Neuro-toxicity & Hepatic-toxicity \\
\hline G1 & 15 & 10 & 12 & 15 & 15 & 5 \\
G2 & 8 & 9 & 5 & 8 & 8 & 2 \\
G3 & 2 & 4 & 2 & 3 & 5 & 1 \\
G4 & 0 & 1 & 0 & 0 & 2 & 0 \\
(\%) & $25 \%$ & $24 \%$ & $19 \%$ & $26 \%$ & $27 \%$ & $8 \%$ \\
\hline
\end{tabular}

Table 4. Univariate analysis of predicators on early failure for the stage III CRC patients in the study.

\begin{tabular}{|c|c|c|c|c|c|}
\hline & \multicolumn{4}{|c|}{ Early failure } & \multirow{3}{*}{$P$ value } \\
\hline & \multicolumn{2}{|c|}{ No $(n=80)$} & \multicolumn{2}{|c|}{ Yes $(n=20)$} & \\
\hline & No. & $\%$ & No. & $\%$ & \\
\hline \multicolumn{6}{|l|}{ Age } \\
\hline$<40$ years & 38 & 47.5 & 9 & 45 & \multirow{2}{*}{0.592} \\
\hline$\geq 40$ years & 42 & 52.5 & 11 & 55 & \\
\hline \multicolumn{6}{|l|}{ Gender } \\
\hline Male & 42 & 40.0 & 10 & 50 & \multirow{2}{*}{0.706} \\
\hline Female & 38 & 60.0 & 10 & 50 & \\
\hline \multicolumn{6}{|l|}{ Site } \\
\hline Colon & 61 & 67.5 & 13 & 65 & \multirow{2}{*}{0.779} \\
\hline Rectum & 19 & 32.5 & 7 & 35 & \\
\hline \multicolumn{6}{|l|}{ Pathology } \\
\hline Adenocarcinoma & 72 & 75 & 14 & 70 & \multirow{2}{*}{0.280} \\
\hline Other variants & 8 & 25 & 6 & 30 & \\
\hline \multicolumn{6}{|l|}{ Grade } \\
\hline Well diff & 18 & 57.5 & 6 & 30 & \multirow{3}{*}{0.229} \\
\hline Mod diff & 24 & 36.25 & 8 & 40 & \\
\hline Poorly diff & 24 & 6.25 & 6 & 30 & \\
\hline \multicolumn{6}{|l|}{ Tumor invasion } \\
\hline $\mathrm{T} 2$ & 9 & 12.5 & 5 & 25 & \multirow{3}{*}{$0.002^{*}$} \\
\hline $\mathrm{T} 3$ & 66 & 80.0 & 4 & 20 & \\
\hline $\mathrm{T} 4$ & 5 & 7.5 & 11 & 50 & \\
\hline \multicolumn{6}{|l|}{ Lymph node status } \\
\hline N1 & 43 & 56.25 & 9 & 45 & \multirow{2}{*}{0.249} \\
\hline $\mathrm{N} 2$ & 37 & 43.75 & 11 & 55 & \\
\hline \multicolumn{6}{|l|}{ CEA } \\
\hline Normal & 53 & 75 & 5 & 25 & \multirow{2}{*}{$0.041^{*}$} \\
\hline Abnormal & 27 & 25 & 15 & 75 & \\
\hline \multicolumn{6}{|l|}{ ERCC1 } \\
\hline Positive & 5 & 12.5 & 16 & 80 & \multirow{2}{*}{$0.004^{*}$} \\
\hline Negative & 75 & 87.5 & 4 & 20 & \\
\hline
\end{tabular}

${ }^{\star}$ Significant difference $(\mathrm{P}<0.05)$. 
Table 5. Binary logistic regression multivariate analysis of predicators on early failure for the stage III CRC patients in the study.

\begin{tabular}{cccc}
\hline & P value & ODR & 95\% CI \\
\hline Variables in the equation & & & \\
$\bullet \quad$ ERCC1 & $0.018^{*}$ & 0.045 & $(1.1-2.4)$ \\
$\bullet \quad$ Tumor invasion & $0.001^{*}$ & 1.42 & $(0.003-0.57)$ \\
$\bullet \quad$ CEA & $0.041^{*}$ & 9.5 & $(0.83-109.2)$ \\
Variables not in the equation & & & \\
Age & 0.843 & - & \\
Gender & 0.889 & - & \\
Site & 0.202 & - & \\
Pathology & 0.700 & - & \\
Grade & 0.579 & & \\
$\mathrm{~N}$ & 0.995 &
\end{tabular}

${ }^{*}$ Significant predictor $(\mathrm{P}<0.05)$.

CRC patients. However, large proportion of patients displays varying levels of treatment resistance, indicating that the therapeutic efficacy has anotableinter-individual variability. Hence, it is important to find biomarkers that might enable the selection of which chemotherapy regimen offers the greatest benefit in an individual patient. Several studies have investigated the influence of ERCC1 in resistance to oxaliplatin compound in CRC patients. Shirota et al. found that low mRNA ERCC1 expression is related to better survival in irinotecan-resistant CRC patients treated with oxaliplatin [15]. Lenz study found that the advanced CRC with high expression of ERCC1 mRNA should not be offered oxaliplatin-based chemotherapy [20]. Li et al. found that ERCC1 expression remained an independent significant prognostic factor for DFS and OS among patients with CRC on oxaliplatin-based chemotherapy [19]. In addition, Opus study found that Patients in the FOLFOX4 arm in the high ERCC1 expression group had shorter PFS, overall survival and a lower response rate compared with those in the low ERCC1 expression group [21] while Ishibashi et al. and Jae et al. also found no significant association between ERCC1 expression and response to chemotherapy [18] [22]. Huang et al. demonstrated that ERCC1 overexpression, was significant predictive factor for early failure. Furthermore, the DFS and OS in CRC patients with ERCC1 overexpression were also prominently shorter than that in CRC patients without ERCC1 overexpression [18]. Similarly, in our study, among patients with stage III CRC, a multivariate logistic regression analysis showed that positive ERCC1 tumor was independent predictor of early treatment failure. Additionally, positive ERCC1 tumors had lower DFS. This finding is consistent with the known function of ERCC1 in DNA repair following platinum therapy. Cancer cells with ERCC1 overexpression may have higher DNA repair capacity that could effectively reduce the anticancer effect of oxaliplatin, leading to poor prognosis of these patients. Moreover, ERCC1 expression often possesses a high DNA-repair capability, and so, upon exposure to oxaliplatin, will un- 
dergo relatively less apoptosis. Apoptosis is also one of the main mechanisms through which platinum compounds exert their antineoplastic activity, so less apoptosis is related to poor therapy efficacy and leading to treatment failure [23]. ERCC1 expression was significantly correlated with histologic grade in our results and this significant correlation was reported in a previous study [24]. However, the interpretation of this result is limited by the small number of patients in our study; other researchers did not report this significant correlation.

\section{Conclusion}

In summary, our findings showed that ERCC1 is an independent predictive factor for early failure and DFS in stage III CRC patients. This supports the hypothesis that ERCC1 may be useful for improving the chemotherapeutic outcome in patients with stage III CRC. When ERCC1 overexpression is present, it seems possible to identify CRC patients who gain the least benefit from the addition of OX to 5-FU. Selecting alternative chemotherapeutic regimens or even adding agents on the basis of pharmacogenetics information may present an innovative strategy. Also, it may help to identify patients who would benefit from intensive follow-up.

\section{References}

[1] Torre, L.A., Bray, F. and Siegel, R.L. (2015) Global Cancer Statistics, 2012. CA: A Cancer Journal for Clinicians, 65, 87. http://dx.doi.org/10.3322/caac.21262

[2] Andre, T., Boni, C. and Mounedji-Boudiaf, L. (2004) Oxaliplatin, Fluorouracil, and Leucovorin as Adjuvant Treatment for Colon Cancer. New England Journal of Medicine, 350, 2343-2345. http://dx.doi.org/10.1056/NEJMoa032709

[3] Hari, D.M., Leung, A.M. and Lee, J.H. (2013) AJCC Cancer Staging Manual 7th Edition Criteria for Colon Cancer: Do the Complex Modifications Improve Prognostic Assessment? Journal of the American College of Surgeons, 217, 181-190.

[4] Mayer, R.J. (2012) Oxaliplatin as Part of Adjuvant Therapy for Colon Cancer: More Complicated than Once Thought. Journal of Clinical Oncology, 30, 3325-3327. http://dx.doi.org/10.1200/JCO.2012.44.1949

[5] Ohlsson, B., Breland, U. and Ekberg, H. (1995) Follow-Up after Curative Surgery for Colorectal Carcinoma. Randomized Comparison with No Follow-Up. Diseases of the Colon \& Rectum, 38, 619-626. http://dx.doi.org/10.1007/BF02054122

[6] Buyse, M. and Piedbois, P. (2001) Should Dukes' B Patients Receive Adjuvant Therapy? A Statistical Perspective. Seminars in Oncology, 28, 20-24. http://dx.doi.org/10.1016/S0093-7754(01)90247-7

[7] Buyse, M., Thirion, P. and Carlson, R.W. (2000) Relation between Tumor Response to First-Line Chemotherapy and Survival in Advanced Colorectal Cancer: A Meta-Analysis. Meta-Analysis Group in Cancer. Lancet, 356, 373-378. http://dx.doi.org/10.1016/S0140-6736(00)02528-9

[8] Grothey, A. and Goldberg, R.M. (2004) A Review of Oxaliplatin and Its Clinical Use in Colorectal Cancer. Expert Opinion on Pharmacotherapy, 5, 2159-2170. http://dx.doi.org/10.1517/14656566.5.10.2159

[9] Vineis, P., Talaska, G. and Malaveille, C. (1996) DNA Adducts in Urothelial Cells: Rela- 
tionship with Biomarkers of Exposure to Arylamines and Polycyclic Aromatic Hydrocarbons from Tobacco Smoke. International Journal of Cancer, 65, 314-316. http://dx.doi.org/10.1002/(SICI)1097-0215(19960126)65:3<314::AID-IJC6>3.0.CO;2-2

[10] Martin, L.P., Hamilton, T.C. and Schilder, R.J. (2008) Platinum Resistance: The Role of DNA Repair Pathways. Clinical Cancer Research, 14, 1291-1295.

http://dx.doi.org/10.1158/1078-0432.CCR-07-2238

[11] Metzger, R., Bollschweiler, E. and Hölscher, A.H. (2010) ERCC1: Impact in Multimodality Treatment of Upper Gastrointestinal Cancer. Future Oncology, 6, 1735-1749. http://dx.doi.org/10.2217/fon.10.140

[12] Yao, A., Wang, Y. and Peng, X. (2014) Predictive Value of Excision Repair Cross-Complementation Group 1 Expression for Platinum-Based Chemotherapy and Survival in Gastric Cancer: A Meta-Analysis. Journal of Cancer Research and Clinical Oncology, 140, 2107-2117. http://dx.doi.org/10.1007/s00432-014-1758-4

[13] Steffensen, K.D., Smoter, M. and Waldstrøm, M. (2014) Resistance to First Line Platinum Paclitaxel Chemotherapy in Serous Epithelial Ovarian Cancer: The Prediction Value of ERCC1 and Tau Expression. International Journal of Oncology, 44, 1736-1744. http://dx.doi.org/10.3892/ijo.2014.2311

[14] Wei, H.B., Hu, J., Shang, L.H. and Zhang, Y.Y. (2012) A Meta-Analytic Review of ERCC1/ MDR1 Polymorphism and Chemosensitivity to Platinum in Patients with Advanced NonSmall Cell Lung Cancer. Chinese Medical Journal, 125, 2902-2907.

[15] Shirota, Y., Stoehlmacher, J. and Brabender, J. (2001) ERCC1 and Thymidylate Synthase mRNA Levels Predict Survival for Colorectal Cancer Patients Receiving Combination Oxaliplatin and Fluorouracil Chemotherapy. Journal of Clinical Oncology, 19, 4298-4304.

[16] Kim, S.H., Kwon, H.C. and Oh, S.Y. (2009) Prognostic Value of ERCC1, Thymidylate Synthase, and Glutathione S-Transferase Pi for 5-FU/Oxaliplatin Chemotherapy in Advanced Colorectal Cancer. American Journal of Clinical Oncology, 32, 38-43. http://dx.doi.org/10.1097/COC.0b013e31817be58e

[17] Common Terminology Criteria for Adverse Events (CTCAE) (2010) Version 4.0.

[18] Huang, M.Y., Tsai, H.L. and Lin, C.H. (2013) Predictive Value of ERCC1, ERCC2, and XRCC1 Overexpresssion for Stage III Colorectal Cancer Patients Receiving FOLFOX-4 Adjuvant Chemotherapy. Journal of Surgical Oncology, 108, 457-464. http://dx.doi.org/10.1002/jso.23422

[19] Li, P., Fang, Y.J. and Li, F. (2013) ERCC1, Defective Mismatch Repair Status as Predictive Biomarkers of Survival for Stage III Colon Cancer Patients Receiving Oxaliplatin Based Adjuvant Chemotherapy. British Journal of Cancer, 108, 1238-1244. http://dx.doi.org/10.1038/bjc.2013.83

[20] Grimminger, P.P., Shi, M. and Barrett, C. (2012) TS and ERCC-1 mRNA Expressions and Clinical Outcome in Patients with Metastatic Colon Cancer in CONFIRM-1 and -2 Clinical Trials. Pharmacogenomics Journal, 12, 404-411. http://dx.doi.org/10.1038/tpj.2011.29

[21] Bokemeyer, C., Kohne, C. and Rougier, P. (2010) Cetuximab with Chemotherapy as First-Line Treatment for Metastatic Colorectal Cancer: Analysis of the CRYSTAL and OPUS Studies according to KRAS and BRAF Mutation Status. Journal of Clinical Oncology, 28, 15 s.

[22] Ishibashi, K., Okada, N. and Ishiguro, T. (2010) The Expression of Thymidylate Synthase (TS) and Excision Repair Complementing-1 (ERCC-1) Protein in Patients with Unresectable Colorectal Cancer Treated with mFOLFOX6 Therapy. Gan to Kagaku Ryoho. Cancer \& Chemotherapy, 37, 2532-2535. 
[23] Seetharam, R.N., Sood, A. and Basu-Mallick, A. (2010) Oxaliplatin Resistance Induced by ERCC1 Up-Regulation Is Abrogated by siRNA-Mediated Gene Silencing in Human Colorectal Cancer Cells. Anticancer Research, 30, 2531-2538.

[24] Xu, G.-H., Li, Y. and Ye, S.-L. (2011) ERCC1 and BRCA1 Expressions in the Tissues of Colorectal Cancer and Its Relationship with Clinical Significance of Platinum-Based Chemotherapy. Fudan University Journal of Medical Sciences, 38, 315-318.

Submit or recommend next manuscript to SCIRP and we will provide best service for you:

Accepting pre-submission inquiries through Email, Facebook, LinkedIn, Twitter, etc. A wide selection of journals (inclusive of 9 subjects, more than 200 journals)

Providing 24-hour high-quality service

User-friendly online submission system

Fair and swift peer-review system

Efficient typesetting and proofreading procedure

Display of the result of downloads and visits, as well as the number of cited articles Maximum dissemination of your research work

Submit your manuscript at: http://papersubmission.scirp.org/ 